Freigegebene Version für den Sammelbanddruck:

Chiapparini, E. \& Kovalova, A. (2015). Konfliktlinien zwischen berufstätigen Jugendlichen und elterlichen Erwartungen bezüglich Freizeitgestaltung. In $S$.

Stövesand \& D. Röh (Hrsg.), Konflikte - theoretische und praktische Herausforderungen für die Soziale Arbeit (S. 140-151). Opladen: Barbara Budrich. 


\title{
Konfliktlinien zwischen berufstätigen Jugendlichen und elterlichen Erwartungen bezüglich Freizeitgestaltung
}

\author{
Emanuela Chiapparini und Anastasiya Kovalova \\ Problemaufriss
}

In der Fachliteratur besteht weitgehender Konsens, dass neben der schulischen Bildung vor allem der Familie und den Peers ${ }^{1}$ eine entscheidende Bedeutung für die Lebensgestaltung von Jugendlichen zukommt (bspw. Harring, 2010; Schröder, 2006). Insbesondere das Zusammensein mit Freunden geben Jugendliche als beliebteste Freizeitbeschäftigung in Befragungen in Deutschland und der Schweiz an (vgl. bspw. Willemse et al., 2010). Dennoch deklarieren Jugendliche in der Sekundarstufe II, dass neben Peers auch die eigene Mutter zu den wichtigsten Bezugspersonen zählt (Chiapparini \& Skrobanek, 2012, S. 14; Gensicke, 2010, S. 227229). Außerdem stehen Jugendliche mit dem Beginn der Berufsausbildung vor großen Veränderungen, seien das beispielsweise die steigende Mobilität und finanzielle Ressourcen, was ebenfalls in die Freizeitgestaltung und damit in den Bedeutungsrahmen der Peers einfließt und noch ungenügend untersucht ist (Chiapparini, 2014).

Gleichzeitig sind Entstrukturierungen von Lebensverläufen (Hurrelmann, 2003) sowie Verjugendlichungen von Erwachsenen zu beobachten, indem keine klare Unterscheidung von strukturkonservativen und reformorientierten Positionen zwischen den Generationen in den westlichen Ländern zu identifizieren ist (Scherr, 2014). Damit sind Dynamiken und Verhältnisse zwischen den Generationen schwerer zu erfassen und scharfe Konfliktlinien fehlen (ebd., S. 31). Ebenso zeigt Liegle (2014) auf, wie in einer gegenwärtigen Zeit zunehmender Komplexität, Undurchsichtigkeit und Orientierungslosigkeit ein Wandel der Generationen stattfindet, indem sich der Zusammenhalt sowie die Unterstützung in Familiengenerationen verstärken und gleichzeitig der Kampf um Anerkennung und Konflikte allgemein abnehmen.

1 Mit Peers werden in diesem Kontext gleichaltrige und sozial gleichgestellte Jugendliche bezeichnet (vgl. bspw. Schröder, 2006). 
Daran anschließend stellt sich die Frage, was genau Jugendliche und Erwachsene, Pädagoginnen und Pädagogen sowie Institutionen unter Konflikten, sozialen Problemen und gesellschaftlichen Erwartungen an Jugendliche verstehen. Der in der Fachliteratur gängige Problembegriff ist normativ geprägt, denn er impliziert ein Zweifaches: Jugendliche machen Probleme, aber haben selber auch solche (Groenemeyer, 2014a, S. 50). So verursachen Jugendliche aus der Perspektive der Erwachsenen zwar Probleme, gleichzeitig wird ihnen aber auch eine herausfordernde Lebensphase zuerkannt und ein Bildungsmoratorium zugesprochen, in dem physische, psychische und soziale Entwicklungen stattfinden sollen.

Schließlich fokussiert der vorliegende Beitrag strukturelle Rahmenbedingungen des Aufwachsens von Jugendlichen und versucht damit, auf soziale Ungleichheiten einzugehen und zwischen jugendspezifischen und gesellschaftsspezifischen Ursachen von sozialen Problemen zu differenzieren (Groenemeyer, 2014b; Scherr, 2003). Der Begriff ,Jugend“ wird sozialisationstheoretisch verwendet, indem Jugendliche als produktiv handelnde Subjekte definiert sind (Mansel \& Hurrelmann, 2003, S. 75), die sich zwischen Selbst- und Fremdbestimmung zu orientieren haben. $\mathrm{Zu}$ den Fremdbestimmungen zählt auch die Erfüllung der elterlichen Erwartungen.

\section{Theoretischer Hintergrund}

Der Begriff Jugend ist von der Erwachsenenwelt abhängig, indem dieser von ihr definiert wird und, wie eingangs gezeigt, als soziales Problem (Groenemeyer, 2014a, S. 56) verstanden werden kann. Mit dem Jugendbegriff sind normative Erwartungen verknüpft. Es ist definiert, „was legitimerweise als jugendliches Verhalten gilt“ (ebd., S. 57). Dazu zählt die Bildung von Peergroups, deren Einflussnahme auf das jugendliche Verhalten aufgrund der ähnlichen biografischen Situation als sehr bedeutend festgehalten wird (Scherr 2010). Andere Befunde belegen, dass die Auswahl der Peers von familiärer Sozialisation geprägt ist (Rieker, 2007) oder dass die Eltern ebenso wichtige Vertrauenspersonen für Heranwachsende in Problemlagen sind wie Peers (Chiapparini \& Skrobanek, 2012, S. 14; Gensicke, 2010, S. 227-229). Darüber hinaus verweisen Forschungsergebnisse zum devianten Verhalten auf die Wichtigkeit der Beziehung zu den Eltern (Baier, 2005), sodass bei einer ausgewogenen Beziehung zu den Eltern und bei eigener ausgeprägter Selbstkontrolle gerin- 
geres normabweichendes Verhalten zu beobachten sei. Zudem beeinflussen belastende Familienverhältnisse die Nicht-Erfüllung gesellschaftlicher und elterliche Erwartungen, indem diese Jugendlichen beispielsweise vermehrt Lehrverträge auflösen (Neuenschwander et al., 2012, S. 220 227).

Des Weiteren wissen wohlwollende Eltern mehr über jugendliche Verhaltensweisen (Stattin \& Kerr, 2000), da der Vertrauenskontext zwischen ihnen und den Jugendlichen die Möglichkeit eröffnet, ein Gespräch auf freiwilliger Basis zu führen. Svensson (2003) wiederum stellt einen signifikanten Zusammenhang zwischen elterlicher Aufsicht und dem jugendlichen Substanzmissbrauch fest. Bei geringerer elterlicher Aufsicht erhöht sich der Drogenmissbrauch bei weiblichen und männlichen Jugendlichen.

Grundsätzlich sind Peers und Peergroups aus der Perspektive der Erziehungsbeauftragten zum einen positiv (Chiapparini, 2014, S. 3) konnotiert, denn „ausserhalb der eigenen Familie können Konfliktstrategien entwickelt, erlernt und eingeübt werden“, so Harring (2007, S. 250) und ebenso Betz (2004). Zum anderen sind sie negativ behaftet (Beinke, 2006), denn Peergroups führen zu Normabweichungen und gesellschaftlichen Desintegrationen. Weiter kann festgehalten werden, dass seit Beginn der Jugendforschung sozial Benachteiligte sich in den Städten kumulieren (Harring, 2014) und sich darüber hinaus die Gruppenaktivität von Jugendlichen auf besonders auffällige Weise in urbanen Gebieten abzeichnet.

In diesem skizzierten theoretischen Hintergrund sind die drei Grundfragen verankert, denen vorliegender Beitrag nachgeht: Welche elterlichen Erwartungen und Konflikte artikulieren urbane berufstätige Jugendliche im Rahmen der Freizeitaktivitäten in ihren Peergroups? Wie deuten sie elterliche Erwartungen? Welche Bewältigungsstrategien präsentieren sich?

\section{Datenlage und methodischer Zugang}

Im Rahmen einer repräsentativen Studie „SoYouth“ ${ }^{2}$ an Maturitäts- und Berufsschulen im Kanton Zürich wurden Jugendliche im Alter von 16 bis 20 Jahren (Sekundarstufe II) zu ihren Freizeitgestaltungen mit einer On-

2 Zum Forschungsprojekt SoYouth und dessen methodischer Anlage siehe weiterführend Chiapparini et al. (2013, S. 5-6). 
lineumfrage befragt. Daran anschließend wurden Gruppendiskussionen (Bohnsack, 1989; 2006) eingesetzt; dies bildet die Datengrundlage dieses Beitrags, mit der kollektive Deutungen zu elterlichen Erwartungen mittels eines offenen und prozesshaften Nachvollzugs rekonstruiert und der einer Handlung zugrundeliegende Sinn aufgedeckt werden können.

Von allen Jugendlichen, die sich nach der Onlinebefragung freiwillig bereit erklärt hatten, an einer Folgeuntersuchung (Gruppendiskussion) teilzunehmen, wurden vier urbane Peergroups aufgrund ihrer kontrastierenden sozialen Lage ausgewählt (vgl. Tabelle 1).

Tabelle 1: Situierung der vier Gruppendiskussionen anhand der sozialen Lage.

\begin{tabular}{|l|l|l|}
\hline $\begin{array}{l}\text { Peergroup mit ungünsti- } \\
\text { ger sozialer Lage }\end{array}$ & Gruppe Sommer & Gruppe Motor \\
\hline Peers der Gruppe & $\mathrm{Aw}^{3}, \mathrm{Bw}, \mathrm{Cw}, \mathrm{Dw}$ & Am, Bm, Cm \\
\hline $\begin{array}{l}\text { Peergroup mit vorteilhaf- } \\
\text { ter sozialer Lage }\end{array}$ & Gruppe Insel & Gruppe Ferien \\
\hline Peers der Gruppe & Aw, Bw, Cm, Dw, Ew, Fw & Aw, Bw, Cm, Dw, Ew \\
\hline
\end{tabular}

Quelle: SoYouth $2012^{2}$

Die soziale Kontrastierung orientiert sich vorerst an strukturellen Kriterien (basierend auf der Sozialhilfequote) und spiegelt sich in den soziodemografischen Angaben der Teilnehmenden aller vier Gruppendiskussionen, die nachträglich eingeholt wurden.

Eine besonders starke soziale Kontrastierung zeigt sich zwischen der Gruppe Sommer und der Gruppe Insel bezüglich ihrer aktuellen Berufsausbildung. Während in der Gruppe Sommer zwei Peers die Lehre abgebrochen haben, eine Peer ohne Abschluss der Sekundarstufe I ein Zwischenangebot besucht und nur eine Peer eine dreijährige Lehre (Restaurationsfachfrau) besucht, absolvieren fast alle Peers der Gruppe Ferien das Gymnasium; nur eine Peer bildet sich als Kauffrau aus. In allen Gruppen gibt es mindestens jemanden, der die Berufsschule besucht.

3 Der zweite Kleinbuchstabe w oder m verweist auf das Geschlecht der Jugendlichen (weiblich, männlich). 
Die Gruppendiskussionen fanden auf Wunsch der Teilnehmenden in einem Raum der offenen Jugendarbeit statt. Entsprechend der Größe der realen Peergroups beteiligten sich drei bis sechs Jugendliche am Gespräch. Da es sich um eine reale Peergroup handelt, weisen die Teilnehmenden einen vergleichbaren Erfahrungshorizont bezüglich Freizeit bzw. „konjunktiven Erfahrungsraum“ (Bohnsack et al., 1995, S. 8-9) auf. Durch offene Erzählaufforderung, wie sie sich als Gruppe kennengelernt haben, sowie durch Beschreibungsaufforderungen von gemeinsamen Aktivitäten liegen selbstlaufende Berichte vor, in denen die Jugendlichen vorwiegend textimmanent Themen wie „Eigen- und Fremdwahrnehmung, Benachteiligungen und Zukunftswünsche sowie Konflikte in der Gruppe“ mit anderen Gruppen und mit Erwachsenen diskutieren. Aufgrund der offenen Erhebungsweise lässt sich das Verhältnis zwischen Erforschtem und Forschendem (Bohnsack, 1993, S. 26-28) als eine Rollenzuschreibung gegenüber der Interviewerin als einer außenstehenden und neutralen Person beschreiben, die man nur einmal sieht und die sich zwischen den Rollen der Peers und Lehrpersonen sowie Eltern positioniert.

Die Tiefeninterpretation des Gesprächsmaterials wurde in Anlehnung an die Dokumentarische Methode nach Bohnsack (2003, S. 563-564) sequenziell und intersubjektiv durchgeführt. Aus den forschungsthematisch relevanten Textpassagen wurden explizite und implizite Wissensbestände zu elterlichen Erwartungen und Konflikten mit Eltern im Rahmen der Freizeitgestaltung der Jugendlichen differenziert.

\section{Befunde}

Jugendliche geben in der Onlinebefragung an, eher wenig soziale Probleme zu haben (vgl. Abb. 1), wobei sie zu Problemen mit den Eltern, mit Geld, mit dem Leben generell, mit Lehrpersonen, Drogen, der Polizei und mit Freunden in den letzten zwei Jahren befragt wurden. Trotz einer vagen elterlichen Kontrolle, die aus den weiter unten aufgeführten Passagen ersichtlich wird, ist der Stellenwert von Problemen mit Drogen bei Jugendlichen nicht hoch. Dieser Befund widerspricht der Annahme von Svensson (2003), dass schwächeres elterliches Monitoring zu einem stärkeren Substanzmissbrauch bei Heranwachsenden führt.

Abb. 1: Subjektive Problemwahnehmung. $\mathrm{N}=2371$ 
„Nun geht es um verschiedene Probleme, die man haben kann. Wie oft hatten Sie in den letzten zwei Jahren Probleme mit ...

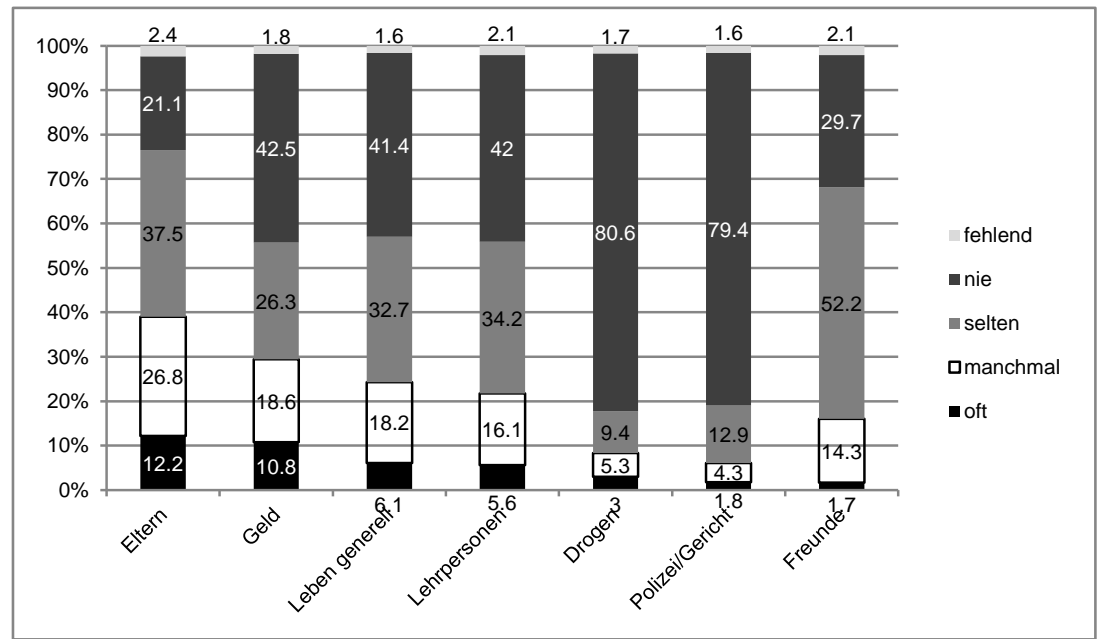

Quelle: SoYouthDat 2012

Des Weiteren geben die Jugendlichen an, am meisten Probleme mit ihren Eltern, mit Geld oder mit ihrem Leben generell zu haben. Interessanterweise geht aus der deskriptiven Analyse der zentralen Vertrauenspersonen hervor (vgl. Abb. 2), dass neben der besten Freundin sowie dem bestem Freund (56\%) die Mutter (56.8\%) gefolgt vom Freundeskreis (43.9\%) eine zentrale Rolle einnimmt, trotz der Tatsache, dass Jugendliche oft Schwierigkeiten mit ihren Eltern haben.

Abbildung 2: Bezugspersonen. $\mathrm{N}=2371$ 


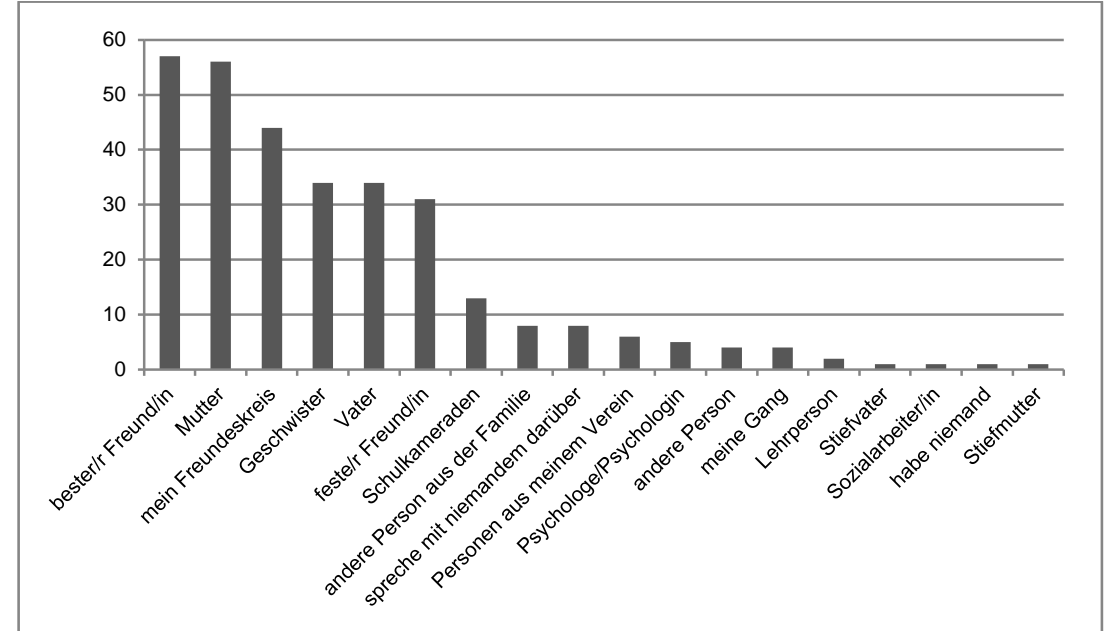

Quelle: SoYouthDat 2012

Zudem geben die Jugendlichen an, die Freizeit größtenteils mit Freunden zusammen zu verbringen (58.7\%) (vgl. Abb. 3). 
Abbildung 3: Personen in der Freizeit. $\mathrm{N}=2371$ „Mit wem verbringen Sie am häufigsten Ihre Freizeit?“

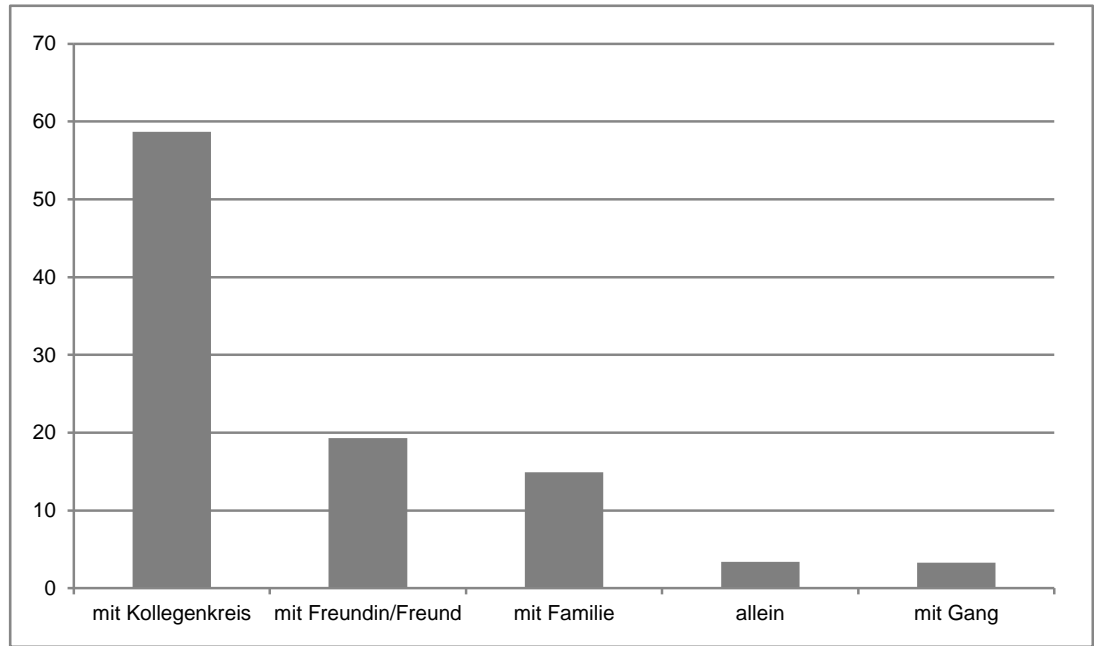

Quelle: SoYouthDat 2012

Damit ist die große Bedeutung von Peergroups einmal mehr unterstrichen, die mit Blick auf die Gruppenstruktur anhand der analysierten Gruppendiskussionen differenziert werden kann. Aus diesen geht hervor, dass sich die Gruppe Sommer und Motor (mit sozial benachteiligtem Hintergrund) geschlossen und regelmäßig treffen. Hingegen stellen die Gruppe Insel und Ferien (mit sozial vorteilhaftem Hintergrund) losere Vergemeinschaftungen dar, die sich punktueller und in unterschiedlichen Mitgliederkompositionen treffen.

Parallel dazu artikulieren die Peers der Gruppe Sommer und Motor klare elterliche Erwartungen anhand von Zeit- und Übernachtungsregeln, die explizit kontrolliert und eher erfolglos sanktioniert werden. In folgender Gesprächspassage der Gruppe Motor wird dies besonders deutlich: ${ }^{4}$

4 Folgende Transkriptionszeichen fanden Verwendung (Bohnsack, 2008, S. 235236): - = Abbruch eines Wortes /Satzes; Nein = laut (in Relation zur üblichen Lautstärke des Sprechers/der Sprecherin; $($ Zahl $)=$ Anzahl der Sekunden, die eine Pause dauert; $L$ = Beginn einer Überlappung; ,...“ = Wörtliche Rede innerhalb der Aussage; @ja@=lachend gesprochenes,,ja“; @(3)@ = Lachen (Dauer in Sek.); $\mathrm{Me}=$ mehrere Personen. 
Cm: (1) Also die Eltern wollen wissen, was wir machen, aber (1) das erfahren sie halt nicht.

Am: Ja sie sagen immer, ja (1): „Schau, er ist immer draussen-“

Bm: L@1@

Cm: Sie wollen auch, dass wir früh zu Hause sind, so: „Um 2 Uhr bist du hier!“ so, aber (1) wir gehen dann um 5 Uhr oder, (1) dann finden sie es halt am nächsten Tag nicht so gut- (1)

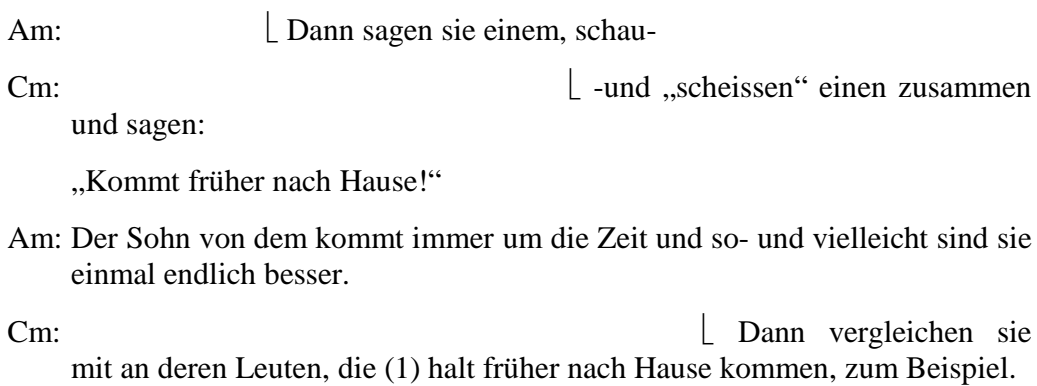

Alle drei Peers der Männergruppe diskutieren intensiv über ihre Freizeitaktivitäten am Wochenende und Cm fügt ein, dass die Eltern die Aufsicht der Heranwachsenden beibehalten wollen. Die Kontrollhaltung der Eltern wird von beiden anderen Freunden geteilt und am Beispiel der Heimkehrzeit am Wochenende, die nicht eingehalten wird, diskutiert. Die Reaktionsmuster der Eltern (Rügen und Vergleiche mit angepassten Jugendlichen) wird von den anderen Peers der Gruppe wahrgenommen, aber in ihren Diskussionen nicht weiter berücksichtigt.

In der Erwähnung des elterlichen Vergleichs zu den anderen Jugendlichen, die eine separate Gruppe bilden, wie dies aus anderen Textpassagen hervorgeht, fällt die Aussage „die einmal endlich besser sind“ auf. Dies dokumentiert, dass die Peergruppe Motor ebenfalls mit dem elterlichen Raster des „Guten und Schlechten“ argumentiert und sich dazu in vielen Freizeitaktivitäten differenziert abgrenzt. Dies veranschaulicht folgende Passage besonders deutlich, indem kulturspezifische Erwartungen der Eltern an die Lebensgestaltung der Heranwachsenden explizit enthalten sind: 
Bm: Und sie („alte Freunde“ der anderen Peergroup) sind halt so (2), sagen wir (1) ehr-lich, so halt (1) extrem hängengeblieben. Sie haben halt noch die alten Vorstellungen man heiratet mit 21, dies das, man macht, ähm, Sachen die einem die Eltern vor-schreiben, man darf ja nicht aus der Reihe tanzen und so-

Am:

dass wir voll die Rebellen sind und so-

L Also es geht nicht darum,

Bm:

L Nein, das nicht.

Am:

$\mathrm{L}$-und man nicht sagen, das macht ihr nicht, das machen wir sofort. (1) Es ist einfach, (1) der Stil, wie sie leben, also (1) es ist einfach (2)

Bm: Es ist einfach eine riesige Fresse aber nichts- (1) also nicht sozusagen leben (3)

Am: $\quad L$ ich sage immer noch- $\quad L$ dahinter

Bm: Also ich finde ich geniesse mein Leben mehr als die, die einfach am Bahn hof hängen und sich krass fühlen-

Am:

$L$-weil sie ein Auto haben. Wir gehen lieber weg (1) weg von diesen Leuten (1) und wirklich mit ruhigen Leute, Leute, die wirklich Party wollen, das Leben geniessen. (3) Tipp top. (1) So Sachen (4).

In dieser Textpassage ist die elterliche und kulturspezifische Erwartung expliziert: Mit 21 Jahren heiraten und den Tätigkeiten nachgehen, welche die Eltern vorschreiben. Später zeigt sich dies in Vorschriften, wie keinen Alkohol konsumieren oder einen Lehrabschluss machen, an welche die Peers der Gruppe Motor sich - zwar verdeckt - nicht halten.

Zudem beschreiben die Mitglieder der Gruppe Motor eine frühere Freundesgruppe als Personen, welche den elterlichen und kulturellen Erwartungen nachgehen und gleichzeitig eine „riesige Fresse haben“ (d.h. viel sagen und sich nicht daran halten). In Abgrenzung zu jener Gruppe charakterisieren sie ihre eigenen Tätigkeiten mit den ausdrucksstarken Verben „leben“ und „genießen“. „Leben“ lässt sich aber nicht mit diesen Leuten, deshalb gehen die Peers der Gruppe Motor von diesen „weg“. Die soziale Distanz wird durch die geografische Distanz verstärkt, indem die Gruppe Motor mit ihrem Auto Ausflüge im Kanton und in Nachbarkantonen unternimmt, andere Leute trifft und etwas erlebt. Damit verbinden sie den Lebensgenuss, bei dem Alkoholkonsum dazugehört. Die Abgrenzung zu jener Gruppe kann in einem weiteren Sinn als Flucht aus dem ei- 
genem Umfeld und Quartier und von den elterlichen Erwartungen gelesen werden.

Während die Gruppen Sommer und Motor die elterlichen Erwartungen der Eltern explizit erwähnen und gleichzeitig ignorieren, werden diese in den Gruppen Insel und Ferien kaum thematisiert und gleichzeitig ernst genommen. Eine Ausnahme bildet eine Gesprächssequenz der Gruppe Insel, in der Dw das Thema der elterlichen Erwartungen anhand von niederschwelligen Vorschriften einführt, die kollektiv geteilt werden:

Dw: Also sie (Eltern) verbieten mir eigentlich nichts gross. Ja, sagen nicht "Das darfst du nicht, das nicht." Sondern, ich soll wieder nach Hause kommen oder sie informieren, wenn ich bei jemandem anderen schlafe. Ja sie müssen einfach ein bisschen infor- miert sein, aber sie kontrollieren mich jetzt überhaupt nicht. Ich bin auch schon ir-gendwie ein bisschen besoffen nach Hause gekommen und die sagen nichts @und ich bin auch umgefallen und so@ (...) Solange ich nicht übertreibe, ist es okay

Cm: Bei mir ist es auch so. (1)

Fw: Also meine sind froh, wenn ich mich nicht übergebe und so. Sonst ist es ihnen egal, $\quad$ wenn ich es nach Hause schaffe. @(1)@

Me: @(1)@

Fw: Ja, es ist ihnen egal.

Ew: Ja, meinen Eltern ist wichtig, dass ich ihnen eine SMS schreibe, wann ich in etwa nach Hause komme. Sonst ist es ihnen eigentlich auch egal.

In dieser Passage wird eine geteilte Gleichgültigkeit der Eltern gegenüber ihrer risikoreichen Freizeitgestaltung dargestellt, die, trotz des hohen und transparenten Alkoholkonsums der Heranwachsenden, nur über die Heimkehrzeit informiert sein wollen oder ihre Heimkehr garantiert haben wollen. Diese Minimalerwartungen finden sich in beiden Gruppen wieder, welche die Peers erfüllen und was ein Erklärungsgrund sein kann, weshalb Konflikte mit Eltern in beiden Gruppengesprächen kein Thema ist.

Schließlich geht aus unterschiedlichen Textstellen hervor, dass zu den Minimalerwartungen der Eltern die Erfüllung der Schulleistungen zählt, womit die Selbstgestaltung der Wochenenden mit Kollegen ohne elterlicher Kontrolle garantiert ist: „Solange ich in der Schule gut bin@(1)@ ist es ihnen eigentlich egal (1)“ (Cm der Gruppe Ferien). Hingegen ist 
schulische Pflichterfüllung in den beiden bezüglich des sozialen Hintergrundes kontrastierenden Gruppen kein Gesprächsthema.

\section{Fazit}

Rekapitulierend belegen sowohl die deskriptiven Analysen als auch die Auswertung der Gruppendiskussionen eine geringe Konfliktwahrnehmung seitens der Jugendlichen. Dieser Befund ordnet sich in die gegenwärtigen Erkenntnisse aus der Generationenforschung (Scherr, 2014; Liegle, 2014) ein. Darüber hinaus geben die befragten Jugendlichen an, mit den Eltern die meisten Konflikte zu führen, was auf dem ersten Blick ein widersprüchlicher Befund bezüglich einem zunehmenden familiären Zusammenhalt (Liegle, 2014) darstellt. Allerdings verweist die vertiefte Analyse der Gruppendiskussionen auf eine differenzierte Erkenntnis hierzu. Denn elterliche Erwartungen sind bei Jugendlichen präsent, weisen aber herkunftsspezifische Tendenzen auf. Während in den sozial benachteiligten Gruppen klare Regeln zu Ausgangszeit und Übernachtungsorten vorliegen, bestehen in den kontrastierenden Gruppen Minimalregeln bezüglich der Informiertheit der Eltern und der Erfüllung schulischer Leistungen. Parallel dazu ignorieren die kontrollierten Heranwachsenden der ersten zwei Gruppen die explizierten Ermahnungen der Eltern und die damit zusammenhängenden Konfliktsituationen. Hingegen meiden die Jugendlichen aus einem sozial vorteilhaften Hintergrund grundsätzlich elterliche Konflikte, indem sie auf die elterlichen Minimalvorschriften und das damit implizite Vertrauensbündnis mit ihren Eltern eingehen. Konflikte mit Eltern werden nur in den zwei sozial benachteiligten Gruppen thematisiert. Die Vermutung liegt nahe, dass elterliche Konflikte in den zwei anderen Gruppen nicht vorkommen oder diese anders angegangen werden. Gleichzeitig kann in allen Gruppen deren Freizeitgestaltung als risikoreich eingestuft werden, insbesondere was den übermäßigen Alkoholkonsum betrifft. Da sich dieser unabhängig von den dokumentierten und nichtdokumentierten Konflikten mit den Eltern präsentiert, besteht an dieser Stelle weiterer Forschungsbedarf. Ebenso lässt sich aus den Befunden schließen, dass Erziehungsstrategien wie elterliche Kontrolle, Minimalvorschriften oder elterlicher Vertrauensvorschuss keinen klaren Einfluss auf den Alkoholkonsum haben, was den Ergebnissen von Svensson (2003) widerspricht. Vielmehr liegt nahe, dass andere Gründe wie Dyna- 
miken von Peergroups oder die Selbstbestimmung der Freizeitgestaltung eine zentrale Rolle spielen.

Daran anknüpfend lassen sich Konsequenzen für die Soziale Arbeit mit Jugendlichen formulieren: Erstens steigt die Bedeutung offener Jugendarbeit und Streetwork, welche Freiräume für Selbstgestaltung und bestimmung ihrer Freizeit anbieten und gleichzeitig eine präventive Funktion einnehmen, indem beispielsweise bei übermäßigem Alkoholkonsum Gespräche und Hilfen angeboten werden. Dabei ist zentral zu verstehen, was die Jugendlichen als soziales Problem verstehen und wo die Grenze zu risikoreichen Freizeitgestaltungen und damit zu gesellschaftlichen Ausschliessungsprozessen vorliegt. Zweitens ist zu empfehlen, Projekte im Rahmen von Peer-Education zu stärken, um die Dynamiken von Peergroups positiv einzusetzen. Ausgehend von einer jugendlichen Konfliktmeidung oder -ignorierung können drittens Weiterbildungsangebote für Eltern und Jugendarbeitende hilfreich sein, damit Reibungsmomente mit Jugendlichen zugelassen und gefördert werden, um Heranwachsenden Diskussions- und Sensibilisierungsplattformen alternative Freizeitgestaltung anzubieten.

\section{Literatur}

Baier, D. (2005): Abweichendes Verhalten im Jugendalter. Ein empirischer Vergleich verschiedener Erklärungsansätze. (German). Deviant Behavior in Adolescence. An Empirical Comparison of Different Theoretical Explanations. (English). In: Zeitschrift für Soziologie der Erziehung \& Sozialisation, 25, 4, S. 381-398.

Beinke, L. (2006): Der Einfluss von Peer Groups auf das Berufswahlverfahren von Jugendlichen. In: Bley N.; Rullmann M. (Hrsg.): Übergang Schule und Beruf. Aus der Praxis für die Praxis - Region Emscher-Lippe. Wissenswertes für Lehrkräfte und Eltern. Recklinghausen: Fiab, S. 249-265.

Betz, T. (2004): Bildung und soziale Ungleichheit. Lebensweltliche Bildung in (Migranten-)Milieus. Arbeitspapier Trier, S. 2-16.

Bohnsack, R. (1989): Generation, Milieu und Geschlecht. Ergebnisse aus Gruppendiskussionen mit Jugendlichen. Biographie und Gesellschaft. Opladen: Verlag Leske + Budrich.

Bohnsack, R. (1993): Rekonstruktive Sozialforschung. Einführung in Methodologie und Praxis qualitativer Forschung (2. Aufl.). Opladen: Verlag Leske + Budrich.

Bohnsack, R. (2003): Qualitative Methode der Bildanalyse. In: Zeitschrift für Erziehungswissenschaft, 6, 2, S. 239-259.

Bohnsack, R. (2006): Das Gruppendiskussionsverfahren in der Forschungspraxis. Opladen: Verlag Barbara Budrich.

Bohnsack, R. (2008): Rekonstruktive Sozialforschung. Einführung in qualitative Methoden (7. Aufl.). Opladen: Verlag Barbara Budrich. 
Bohnsack, R.; Loos, P.; Schäffer, B.; Städtler K.; Wild, B. (1995): Die Suche nach Gemeinsamkeit und die Gewalt der Gruppe. Hooligans, Musikgruppen und andere Jugendcliquen. Opladen: Verlag Leske + Budrich.

Chiapparini, E. (2014): Schulische Regelbrüche aus der Sicht jugendlicher Peergroups. Schulpädagogik-heute, 5, 9, S. 1-16.

Chiapparini, E.; Skrobanek, J. (2012): Engagement- und Partizipationsformen von Jugendlichen. Eine Vollerhebung unter Oberstufenschülerinnen und -schülern in der March. Forschungsbericht. Zürich: Universität Zürich.

Chiapparini, E.; Hasanmetaj, B.; Kuglstatter, V.; Skrobanek, J.; Zejnuni, K. (2013): Jugenddelinquenz im Kanton Zürich. Handreichung Nr. 3 der Forschungsgruppe SoYouth. Zürich: Universität Zürich.

Gensicke, T. (2010): Wertorientierungen, Befinden und Problembewältigung. In: Shell Deutschland Holding (Hrsg.): Jugend 2010. Eine pragmatische Generation behauptet sich. 16. Shell Jugendstudie. Frankfurt a. M.: Fischer Taschenbuch Verlag, S. 187-242.

Groenemeyer, A. (2014a): Jugend im Problemdiskus - Probleme im Jugenddiskurs. Was bedeutet das Reden über Jugend? In: Groenemeyer, A. (Hrsg.): Jugend als soziales Problem - Probleme der Jugend? Diagnosen, Diskurse und Herausforderungen. Jugendforschung. Weinheim: Beltz Juventa, S. 50-75.

Groenemeyer, A. (2014b): Jugend als soziales Problem - Probleme der Jugend? Diagnosen, Diskurse und Herausforderungen. Jugendforschung. Weinheim: Beltz Juventa.

Harring, M. (2007): Informelle Bildung. Bildungsprozesse im Kontext von Peerbeziehungen im Jugendalter. In: Harring M.; Rohlfs C.; Palentien C. (Hrsg.): Perspektiven der Bildung. Kinder und Jugendliche in formellen, nicht-formellen und informellen Bildungsprozessen. Wiesbaden: VS Verlag für Sozialwissenschaften, S. 237-258.

Harring, M. (2010): Freizeit, Bildung und Peers - informelle Bildungsprozesse im Kontext heterogener Freizeitwelten und Peer-Interaktionen Jugendlicher. In: Harring M.; Böhm-Kasper O.; Rohlfs C.; Palentien C. (Hrsg.): Freundschaften, Cliquen und Jugendkulturen. Peers als Bildungs- und Sozialisationsinstanzen. Wiesbaden: VS Verlag für Sozialwissenschaften, S. 21-59.

Harring, M. (2014): Freizeitaktivitäten der Peers in städtischen und ländlichen Regionen. In: Köhler, S.-M.; Krüger, H.H.; Pfaff, N. (Hrsg.): Handbuch Peerforschung. Opladen: Verlag Barbara Budrich.

Hurrelmann, K. (2003): Der entstrukturierte Lebenslauf. In: Zeitschrift für Soziologie der Erziehung und Sozialisation, 23, 2, S. 115-126.

Liegle, L. (2014): Generationenbeziehungen - Universale Verbreitung und kulturspezifische Prägung. In: Faas, St.; Zipperle, M. (Hrsg.): Sozialer Wandel. Herausforderungen für Kulturelle Bildung und Soziale Arbeit. Wiesbaden: Springer Fachmedien, S. 51-59.

Mansel, J.; Hurrelmann, K. (2003): Jugendforschung und Sozialisationstheorie. Über die Möglichkeiten und Grenzen der Lebensgestaltung im Jugendalter. In: Mansel, J., Griese, H.; Scherr, A. (Hrsg.): Theoriedefizite der Jugendforschung: Standortbestimmung und Perspektiven. Weinheim: Juventa-Verl, S. 7590.Neuenschwander, M. P.; Gerber, M.; Frank, N.; Rottermann, B. (2012): Schule und Beruf. Wege in die Erwerbstätigkeit. Wiesbaden: VS Verlag für Sozialwissenschaften. 
Rieker, P. (2007): Problemlösung in Familien und Peergroup. In: Zeitschrift für Soziologie der Erziehung und Sozialisation, 27, 3, S. 304-319.

Scherr, A. (2003): Konturen einer genuin soziologischen Jugendforschung. In: Mansel, J.; Griese, H. M.; Scherr, A. (Hrsg.): Theoriedefizite der Jugendforschung: Standortbestimmung und Perspektiven. Jugendforschung. Weinheim: Juventa, S. 49-66.

Scherr, A. (2010): Cliquen/informelle Gruppen: Strukturmerkmale, Funktionen und Potentiale. In: Harring, M.; Böhm-Kasper, O.; Rohlfs, C.; Palentien, C. (Hrsg.): Freundschaften, Cliquen und Jugendkulturen. Peers als Bildungs- und Sozialisationsinstanzen. Wiesbaden: VS Verlag für Sozialwissenschaften, S. 73-90.

Scherr, A. (2014): Jugend als soziale Kategorie. Oder: Warum Jugend keine Gruppe und auch kein soziales Problem ist. In: Groenemeyer, A. (Hrsg.): Jugend als soziales Problem - Probleme der Jugend? Diagnosen, Diskurse und Herausforderungen. Jugendforschung. Weinheim: Beltz Juventa, S. 29-49.

Schröder, A. (2006): Cliquen und Peers als Lernort im Jugendalter. In: Rauschenbach, T.; Düx, W.; Sass, E. (Hrsg.): Informelles Lernen im Jugendalter. Vernachlässigte Dimensionen der Bildungsdebatte. Beiträge zur Kinder- und Jugendhilfeforschung. Weinheim: Juventa, S. 173-202.

Stattin, H.; Kerr, M. (2000): Parental monitoring: a reinterpretation. Child Development, 71, 4, S. 1072-1085.

Svensson, R. (2003): Gender Differences in Adolescent Drug Use. In: Youth \& Society, 34, 3, S. 300-329.

Willemse, I.; Waller, G.; Süss, D. (2010). James. Jugend, Aktivitäten, Medien - Erhebung Schweiz / Ergebnisbericht zur James-Studie 2010. Zürich: ZHAW. Departement Angewandte Psychologie. 